\title{
Geographic-based Routing in Smart Grid's Neighbor Area Networks
}

\author{
Quang-Dung Ho, Gowdemy Rajalingham, Tho Le-Ngoc \\ Department of Electrical and Computer Engineering, McGill University, Montreal, Quebec, Canada
}

Correspondence: Quang-Dung Ho, quang.ho@mcgill.ca

Manuscript communication: received 20 November 2013, accepted 9 January 2014

\begin{abstract}
Neighbor area network (NAN), also known as smart meter communication network, is one of the most important segments of smart grid communications network (SGCN). This paper studies the performance of greedy perimeter stateless routing (GPSR), a representative implementation of geographic-based routing class, in the NAN scenario and investigates the feasibility of this routing protocol in supporting SG applications. Specifically, packet transmission delay and reliability of GPSR in an IEEE 802.15.4-based wireless mesh NAN with practical system parameters are measured by simulations. The results show that, at the data rate required for conventional SG applications including smart metering, real-time pricing and demand response, the delay can always be maintained below $70 \mathrm{~ms}$ (in 95th-percentile perspective) while packet delivery ratio is higher than $90 \%$. However, due to that fact that more advanced applications that require information exchange at higher rates and more stringent delays are emerging in SG, the performance of GPSR in NAN scenarios using radio technologies that can support higher loads and/or larger network scales needs to be studied.
\end{abstract}

Keywords- Smart grid communication network (SGCN), neighbor area network (NAN), wireless routing, greedy perimeter stateless routing (GPSR).

\section{INTRODUCTION}

The electrical grid has not been innovated over the last century. Utility companies have had to send workers out to collect data required for operating and maintaining the grid. For example, the workers read meters, measure power quality, search for failed power lines or broken equipment. Most of devices used to generate and deliver electricity have not been automated and computerized. The aging of the electrical grid causes incidences of electricity shortages, power quality problems, rolling blackouts, electricity price spikes. Additionally, there has been increased demands on electrical energy and environmental issues. These issues and demands are the driving force behind the need for smart grid (SG). By definition, SG "is an automated, widely distributed energy delivery network characterized by a two-way flow of electricity and information, capable of monitoring and responding to changes in everything from power plants to customer preferences to individual appliances" [1]. It can monitor, protect and automatically optimize the operation of its interconnected elements including central and distributed power plants, energy storage stations, transmission and distribution networks, industrial and building automation systems, end-user thermostats, electric vehicles, appliances and other household devices. In essence, the primary objectives of SG are to allow utilities to generate and distribute electricity efficiently and to allow consumers to optimize their energy consumption.

It is critically important to note that the key to achieving these potential values of SG is to successfully

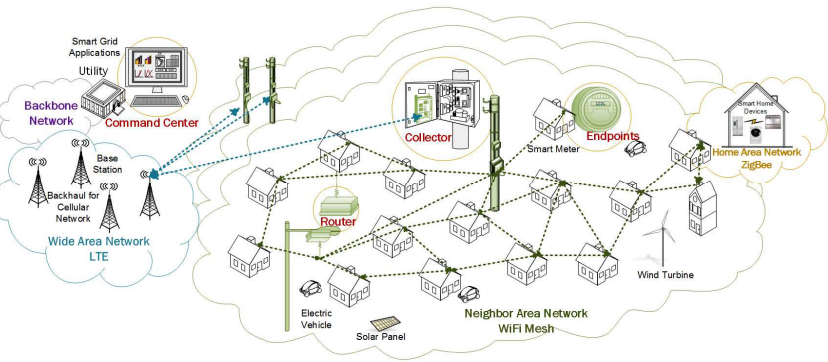

Figure 1. An illustrative implementation of SGCN.

build a smart grid communications network (SGCN) that can support all identified SG functionalities including advanced metering infrastructure (AMI), demand response (DR), electric vehicles, wide-area situational awareness (WASA), distributed energy resources and storage, distribution grid management, etc. [2]. Figure 1 shows an illustrative implementation of SCGN which consists of three segments: home area network (HAN), neighbor area network (NAN) and wide area network (WAN). Among these segments, NAN has been attracting most of concerns from both academia and industry since it is involved in gathering a huge volume of various types of data and distributing important control signals between millions of smart meters (SMs) installed at customer premises and utility's control centers. It enables primarily important SG applications including AMI, DR, distributed energy resources and storage management, etc. There are various wired and wireless communications technologies that can be used to implement NANs such as 


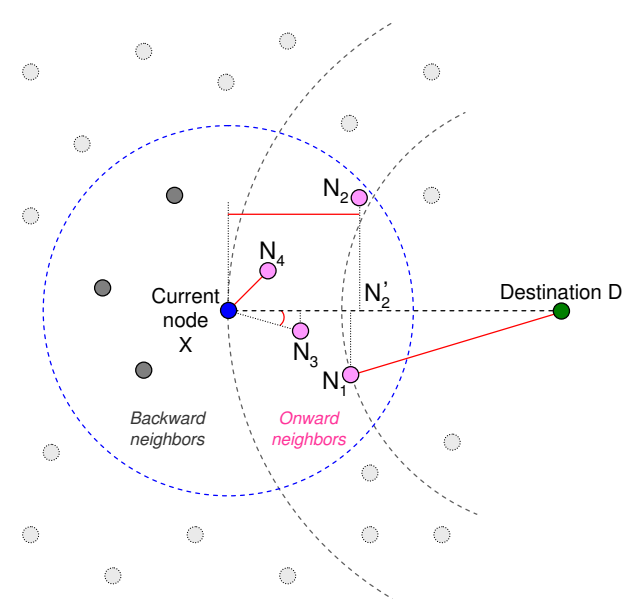

Figure 2. Examples of different GF strategies: $\left(\mathrm{N}_{1}\right)=$ shortest geographical distance, $\left(\mathrm{N}_{2}\right)=$ MFR, $\left(\mathrm{N}_{3}\right)=$ compass routing and $\left(\mathrm{N}_{4}\right)$ $=$ NFP.

broadband via the telephone lines or cable services, power line communications (PLC), wireless cellular and wireless mesh networks (WMNs). Each technology has its own advantages and disadvantages in terms of deployment/maintenance/operation costs, coverage, communication reliability, latency and security, etc. [36]. As illustrated in Figure 1, for the NAN segment, SMs are connected to each other using WMNs that require low deployment, operational and maintenance costs. Data from each cluster of SMs is collected at a data aggregation point (DAP) and then relayed to $3 \mathrm{G} / 4 \mathrm{G}$ cellular networks that have a very wide coverage while offering a high data rate and low latency. Wireless mesh NAN is selected for the study in this paper. Besides, since IEEE 802.15.4 is a mature wireless sensor network technology that can provide radio connectivity at low costs, it is assumed to be used for NANs.

Routing protocol is one of the key factors that determine the system performance of WMNs. As a result, wireless mesh routing protocols for NANs are the focused points of a large number of researches in the area of SG over the last few years [3, 6-16]. Geographicbased routing (GEO) protocols have appeared to be a very good candidate protocol for NANs due to their inherent simplicity and distributed operation. The simplicity results in lightweight implementation and low overhead requirement. The distributed operation leads to ease of network expansion since there is no need to maintain a network graph. A new node can join the network by locally exchanging information with existing nodes in its vicinity. Another important advantage of GEO is that it fully exploits the location information of NAN devices (e.g., SMs, DAPs). However, it is observed that this kind of routing protocol has not been extensively studied in existing work.

This paper attempts to provide a detailed and quantitative evaluation on the performance of greedy perimeter stateless routing (GPSR), a representative form of

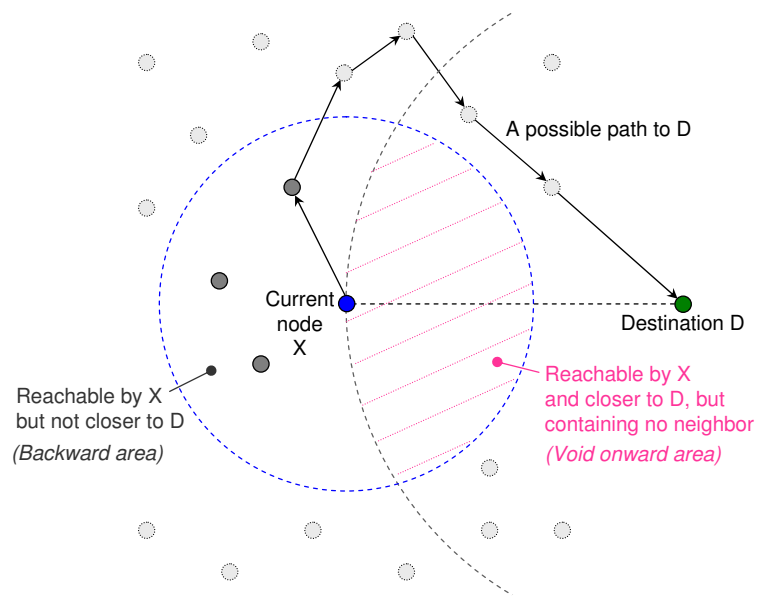

Figure 3. An example of void area: there does not exist any node (within the transmission range of node $\mathrm{X}$ ) closer to destination $\mathrm{D}$.

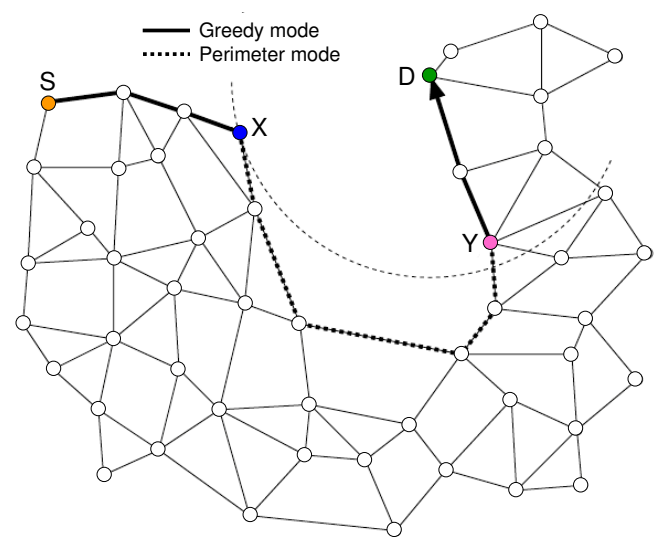

Figure 4. An example of GPSR: the combination of greedy and perimeter modes to circumnavigate the void area.

GEO, in the NAN scenario ${ }^{1}$. Two key performance metrics, i.e., transmission latency and packet delivery ratio, are investigated. The results reveal the effects of transmission and medium access control parameters to the operation and performance of wireless mesh NANs. They also verify the feasibility of GPSR for NANs. The remainder of this paper is organized as follows. Section 2 summarizes main ideas and operations of GPSR. Sections 3 and 4 presents simulation setup and results, respectively. Finally, Section 5 concludes this paper.

\section{An Overview of GPSR}

GEO protocols basically route the traffic based on the knowledge of a node's position together with those of its neighbors and the sink node. An extensive survey of existing work dealing with GEO is presented in [17]. Greedy forwarding (GF) is the simplest implementation of GEO. When a node receives a message, it relays the message to its neighbor geographically closest to the sink, as illustrated in Figure 2. Alternatively, one

\footnotetext{
${ }^{1}$ Parts of this paper were presented in "Performance and applicability of geographic-based routing in smart grid's neighbor area networks" published by the International Conference on Advanced Technologies for Communications (ATC'2013), Oct., 2013.
} 
Table I

Simulation Model and Parameters

\begin{tabular}{|l|l|l|}
\hline \multirow{2}{*}{ PHY Layer } & Standard & IEEE 802.15.4 \\
\cline { 2 - 3 } & Frequency band & $2.4 \mathrm{GHz}$ \\
\hline MAC Layer & Standard & IEEE 802.15.4 \\
\cline { 2 - 3 } & Beacon & Disabled \\
\cline { 2 - 3 } & ACK & Enabled, 11 bytes \\
\cline { 2 - 3 } & Max. retransmissions & 2 \\
\cline { 2 - 3 } & Back-off procedure & Exponential \\
\cline { 2 - 3 } & Back-off exponent range & $(5,8)$ \\
\hline Wireless & Path-loss & $\alpha=2.7$ \\
\cline { 2 - 3 } Channel & Shadowing & Log-normal, $\sigma=7.4$ \\
\hline
\end{tabular}

can consider another notion of progress, namely the projected distance on the source-destination-line, i.e., most forwarding progress within radius (MFR), the minimum angle between neighbor and destination, i.e., compass routing, or nearest with forwarding progress (NFP). The advantage of GEO protocols is that it can achieve network wide routing while maintaining only neighborhood information at each node, hence significantly reducing signaling overheads and the complexity of the routing solution. The fact that locations of NAN devices are fixed and accurately known promotes GEO protocols as one of promising solutions for NANs.

It is noted that GF may lead into a void area where there is no neighbor closer to the destination, as visualized in Figure 3. GPSR uses GF to forward packets to nodes that are always progressively closer to the destination. In regions of the network where such a greedy path does not exist, GPSR recovers by forwarding in perimeter mode, in which a packet traverses successively closer faces of a planar subgraph of the radio network connectivity graph, until reaching a node closer to the destination, where greedy forwarding resumes. An example of GPSR is given in Figure 4 which shows how perimeter is used to circumnavigate the void area. Detailed descriptions of GPSR can be found in [18]. Existing work shows that, compared to the conventional GF, GPSR can improve the packet delivery ratio [19].

\section{Simulation Setup}

A discrete event network simulation platform is used for this study. Non-beacon IEEE 802.15.4 medium access control (MAC) and physical (PHY) layers are implemented in each node. The wireless channel is modeled with simple path-loss (with path-loss exponent $\alpha$ ) and log-normal shadowing $X_{\sigma}$ (with standard deviation $\sigma)$ at independent intervals of $1 \mathrm{~ms}$, as shown below:

$$
P_{\mathrm{R}}[\mathrm{dBm}]=P_{\mathrm{T}}[\mathrm{dBm}]+10 \log _{10}\left(\frac{\lambda^{2}}{16 \pi^{2} d^{\alpha}}\right)-X_{\sigma},
$$

where $P_{\mathrm{T}}$ and $P_{\mathrm{R}}$ are the transmitted and received radio power, respectively; $\lambda$ and $d$ are the wavelength and the transmitter-receiver distance, respectively. The values for path-loss and shadowing parameters are taken from the National Institute of Standards and Technology's SG Priority Action Plan 2 [20], as shown in Table I.

Besides, all nodes are assumed to be homogeneous. Details of the radio interface are chosen to match the

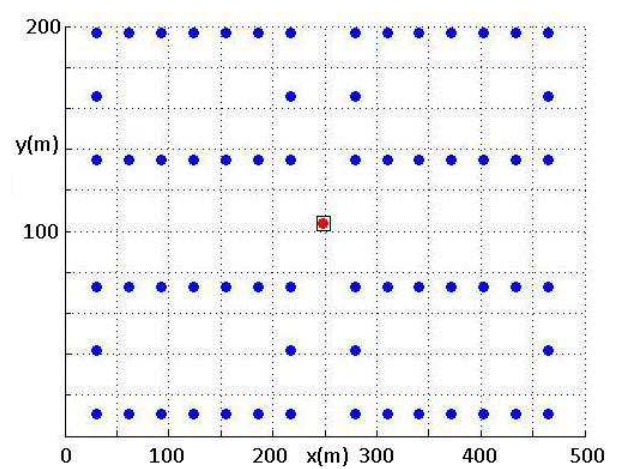

Figure 5. NAN topology with a density of $800 \mathrm{SMs} / \mathrm{km}^{2}$ and a ratio of 64 SMs/DAP [20].

specifications of the Texas Instruments CC2530 system on chip. Specifically, half-duplex communications is assumed with a radio turnaround time $\left(T_{\mathrm{TA}}\right)$ of 0.192 ms. As for collision avoidance, exponential back-offs with $0.32 \mathrm{~ms}$ slots is implemented. The total back-off time $\left(T_{\mathrm{BO}}\right)$ is the product of the number of back-off slots and the slot time. Additionally, the back-off exponent is incremented by 1 (up to a maximal value) for each successive back-off. Furthermore, the inter-frame spacing $\left(T_{\mathrm{IFS}}\right)$ is set to be $0.192 \mathrm{~ms}$. Each acknowledgement (ACK) is 11 bytes in length and its transmission time $\left(T_{\mathrm{ACK}}\right)$ is $0.352 \mathrm{~ms}$. Detailed simulation parameters are listed in Table I.

Periodic traffic is generated in the uplink convergecast direction (from SMs to DAP). For the data rate, a weighted average of the monitoring application traffic is considered. In particular, when taking into consideration the size of the data frame and the frequency of updates, the data rate specified in [20] is equivalent to packets of 79 bytes at a rate of $2.4624 \times 10^{-3}$ packets/node/s.

In order to model the real-life arrangement of houses within a representative deployment of NANs, a gridlike topology is assumed where roads are represented by voids. In detail, a suburban density of $800 \mathrm{SMs} / \mathrm{km}^{2}$ and a ratio of 64 SMs/DAP specified in [20] are chosen, as shown in Figure 5. The performance of the network is analyzed based on path characteristic, transmission latency as well as packet deliver ratio (PDR). The path characteristic is gauged with the average hop count. The average and 95th percentile of transmission delay are investigated. PDR is defined as the percentage of transmitted packets that are received and successfully decoded by DAP. Packet transmission delay is evaluated as follows:

$$
D(n)=T_{\mathrm{BO}}+T_{\text {frame }}(n)+T_{\mathrm{TA}}+T_{\mathrm{ACK}}+T_{\mathrm{IFS}} .
$$

It can be seen from Eq. (2) that, for a packet of 79 bytes, $T_{\text {frame }}$ is $2.53 \mathrm{~ms}$. Therefore, with no backoffs or retransmissions, the delay is roughly 3.266 ms. However, since back-offs and retransmissions are expected, the average and the 95th percentile of the transmission delay are analyzed. In order to assess which SG applications can be supported, the latency 
Table II

SG Application Latency Guidelines [21]

\begin{tabular}{|l|l|}
\hline Application & Latency \\
\hline Demand response (DR) & $2 \sim 5 \mathrm{~min}$. \\
\hline Remote connect/disconnect & $2 \sim 5 \mathrm{~s}$ \\
\hline $\begin{array}{l}\text { Advanced metering infrastructure (AMI), } \\
\text { Real-time pricing, Meter data management, } \\
\text { Outage management }\end{array}$ & $2 \mathrm{~s}$ \\
\hline AMI network management & $1 \sim 2 \mathrm{~s}$ \\
\hline In-home displays & $300 \mathrm{~ms} \sim 2 \mathrm{~s}$ \\
\hline Emergency response & $500 \mathrm{~ms}$ \\
\hline Sychrophasors & $10 \sim 100 \mathrm{~ms}$ \\
\hline $\begin{array}{l}\text { Distribution automation protection event no- } \\
\text { tification }\end{array}$ & $2 \sim 10 \mathrm{~ms}$ \\
\hline
\end{tabular}

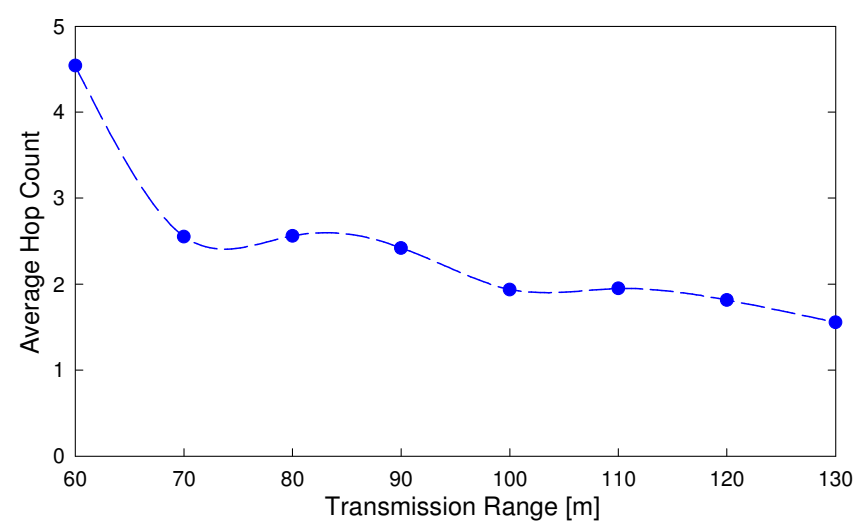

Figure 6. Average hop count versus transmission ranges.

estimates for most typical SG applications are shown in Table II as a reference.

\section{Simulation Results}

In order to determine the feasibility of GPSR for an IEEE 802.15.4-based NAN, the network depicted in Figure 5 is simulated for system parameters estimated in [20]. The transmission range of the radio is swept as the network density, offered load and SM-to-DAP ratio are held constant. Theoretically, given the convergecast nature of the uplink traffic, as the transmission range is increased, an increase in the set of nodes within the direct transmission range of each transmitter should occur. In addition, since the network is homogeneous, DAP experiences the same tendency. This implies that as transmission range is increased, the set of critical nodes (one hop away from DAP) monotonically increases. Therefore, on average, the path length (in terms of number of hops) decreases. Additionally, since the data rate is held constant, a larger set of critical nodes alleviates their load and lessens the bottleneck effect of the convergecast traffic. This load balancing effect should result in less channel contention and thus reduce the need for back-off and retransmission and thereby reducing the average transmission delay. Therefore, as the transmission range is increased, it is expected to see (i) smaller average hop count, (ii) lower delay and (iii) higher PDR.

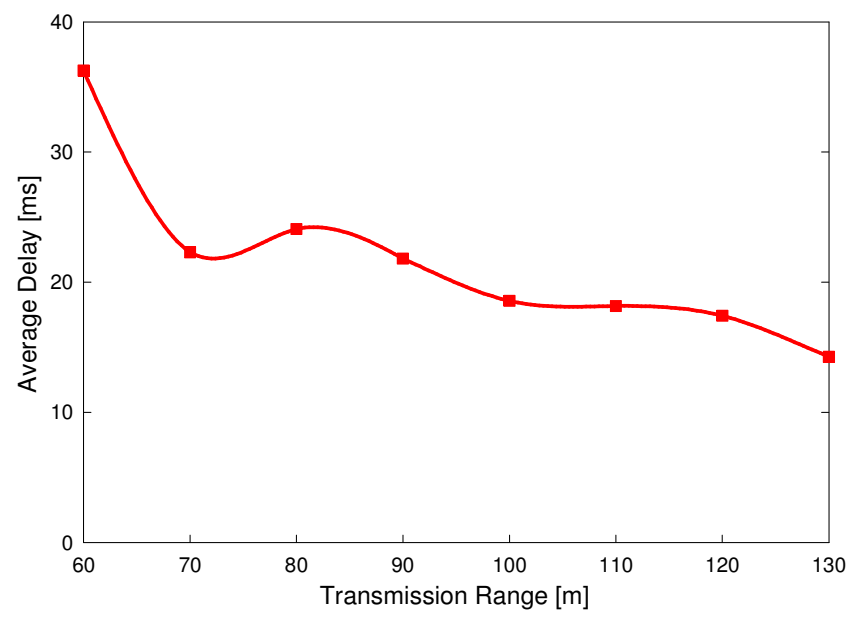

Figure 7. Average delay versus transmission ranges.

\subsection{Routing Path Length}

As the transmission range is increased, shorter and more reliable paths can be achieved. Specifically, as shown in Figure 6, the average hop count drops from 4.5 hops at $60 \mathrm{~m}$ transmission range down to roughly 1.55 hops at $130 \mathrm{~m}$ transmission range. Therefore, since the neighbor sets increase with transmission range, a larger proportion of packets reach their destinations within 1 to 2 hops. It is noted that the curve flattens out for a few regions (e.g., $70 \mathrm{~m}$ to $90 \mathrm{~m}$ transmission range or $100 \mathrm{~m}$ to $130 \mathrm{~m}$ transmission range). This phenomenon can be explained by the network connectivity since the average nodal distance is roughly $30 \mathrm{~m}$ (the network connectivity will remain about constant for roughly $30 \mathrm{~m}$ ). Thus, as expected, given the load balancing effect of the increased critical node set, with higher transmission range, packets are routed over shorter paths to reach the destination.

\subsection{Transmission Delay}

When considering the average transmission delay, the same overall trends from the average hop count can be seen. In particular, as plotted in Figure 7, the average delay reduces from $36 \mathrm{~ms}$ at $60 \mathrm{~m}$ transmission range down to $14 \mathrm{~ms}$ at $130 \mathrm{~m}$ transmission range. Once again, the flat regions over transmission ranges for which no significant change in network connectivity are observed. Furthermore, the overall decreasing trend is explained by the load balancing notion since the main cause for longer delays is the collision avoidance measures. When fewer numbers of backoffs and retransmissions are required, the transmission delay approaches the minimum value calculated in the previous section since a greater number of packets can reach the destination without the added delays from the collision avoidance procedure. However, increasing the transmission range also results in stronger radio signal interference among nodes. Therefore, the performance cannot be further improved by continually increasing the transmission range.

For a more in-depth investigation, 95th percentile of transmission delay is plotted in Figure 8. As can be 


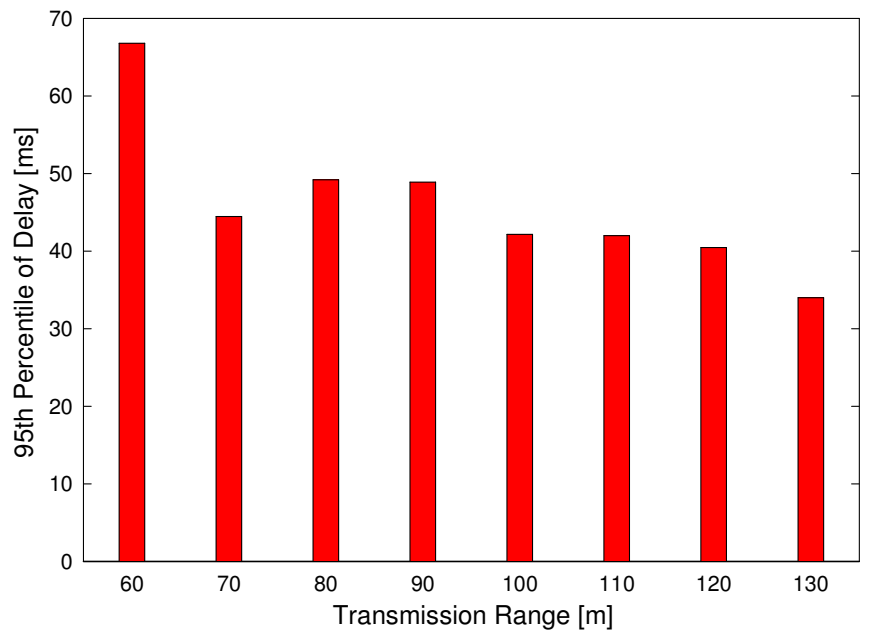

Figure 8. 95th percentile of delay versus transmission ranges.

seen, there is a decrease from $67 \mathrm{~ms}$ at $60 \mathrm{~m}$ transmission range down to $34 \mathrm{~ms}$ at $130 \mathrm{~m}$ transmission range. Additionally, when comparing 95th percentile of delay given by the simulated network against typical delay requirements in Table $\mathrm{II}$, the range of $34 \mathrm{~ms}$ to $67 \mathrm{~ms}$ could indeed handle a great number of SG applications. Predominantly, this result is favorable for the monitoring applications as opposed to the control applications that have stringent requirements.

\subsection{PDR}

Satisfying latency requirements alone is insufficient and the transmission reliability must also be considered. As a result, PDR is plotted in Figure 9. As can be seen, the reliability of packet transmission is relatively high, ranging from $90 \%$ to $95 \%$ for the simulated NAN scenario. However, it is important to note that this reliability is obtained at the base data rate suggested in [20] (which is quite low compared to the physical data rate of IEEE 802.15.4 radio). In other words, only low-bandwidth monitoring applications such as AMI, DR and outage management can be supported. Another observation is that when the transmission range is increased (by using a higher transmission power level), PDR is improved, however the improvement is insignificant (only around $5.7 \%$ when the range is doubled from $60 \mathrm{~m}$ to $120 \mathrm{~m}$ ). This can be explained due to the fact that a longer transmission range may help to reduce the number of communication hops that a packet needs to traverse to reach its destination but at the same time increase per-hop transmission counts caused by a higher level of interference.

\section{Conclusions}

This paper studies the potential of greedy perimeter stateless routing (GPSR) protocol in neighbor area networks (NANs) of smart grid (SG) due to its low complexity, distributed operation and scalability. The performance of GPSR in an IEEE 802.15.4-based NAN with system parameters specified by National Institute of

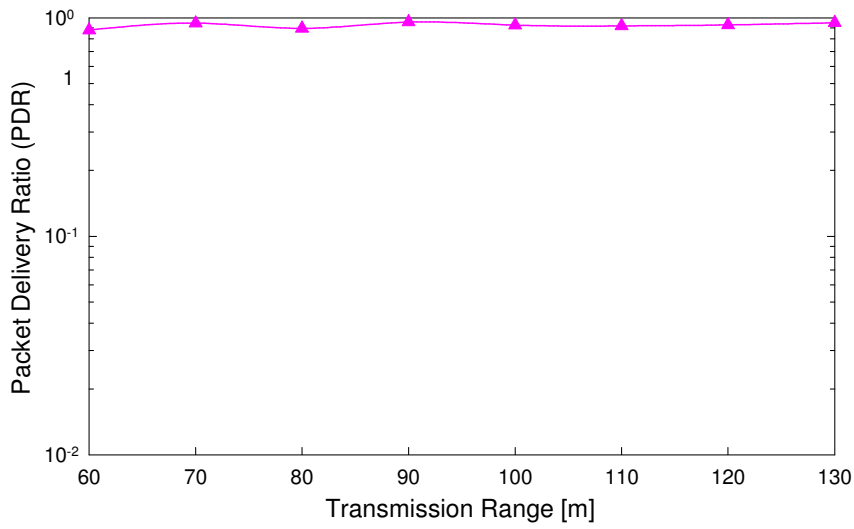

Figure 9. PDR versus transmission ranges.

Standards and Technology is measured by simulations. In details, the achievable latency and reliability are investigated and compared with the current estimates for various SG applications. The results demonstrate that a variety of conventional SG applications such as smart metering, real-time pricing, demand response, etc., can indeed be supported. However, new applications (e.g., advanced distribution automation, fault detection and restoration and so on) that require information exchange at higher rates and more stringent delays are emerging in SG. Therefore, the performance of GPSR in NANs using other radio technologies that can support high-speed and low-latency network connectivity needs to be studied. Furthermore, analysis of downlink traffic, convergecasting with multiple DAPs and load balancing still remain to be explored.

\section{ACKNOWLEDGMENT}

This work is partially supported by the Natural Sciences and Engineering Research Council (NSERC) and industrial and government partners, through the NSERC smart microgrid research network (NSMGNet).

\section{REFERENCES}

[1] Draft Guide for Smart Grid Interoperability of Energy Technology and Information Technology Operation With the Electric Power System (EPS), and End-Use Applications and Loads, IEEE P2030 Std., 2011.

[2] "Communications requirements of smart grid technologies," US's Department of Energy, Tech. Rep., Oct 2010.

[3] Q.-D. Ho, Y. Gao, and T. Le-Ngoc, "Challenges and research opportunities in wireless communications networks for smart grid," IEEE Wireless Communications, pp. 89-95, Jun. 2013.

[4] C.-W. Chao, Q.-D. Ho, and T. Le-Ngoc, "Challenges of power line communications for advanced distribution automation in smart grid," in 2013 IEEE PES General Meeting, Jul. 2013.

[5] Q.-D. Ho, C.-W. Chao, M. Derakhshani, and T. LeNgoc, "An analysis on throughput and feasibility of narrow-band power line communications in advanced distribution automation scenarios," in IEEE International Conference on Communications (ICC'2014), Jun. 2014. 
[6] N. Saputro, K. Akkaya, and S. Uludag, "A survey of routing protocols for smart grid communications," Computer Networks, vol. 56, no. 11, pp. 2742 - 2771, 2012.

[7] M. Nucolone, "Stability analysis of the delays of the routing protocol over low power and lossy networks," Master's thesis, KTH Electrical Engineering, 2010.

[8] D. Wang et al., "RPL based routing for advanced metering infrastructure in smart grid," Mitsubishi Electric Research Laboratories, Tech. Rep. TR2010-053, Jul 2010.

[9] J. Tripathi, J. C. de Oliveira, and J. P. Vasseur, "Applicability study of RPL with local repair in smart grid substation networks," in Proc. the First IEEE International Conference on Smart Grid Communications (SmartGridComm), Oct 2010, pp. 262-267.

[10] P. Kulkarni et al., "A self-organising mesh networking solution based on enhanced RPL for smart metering communications," in Proc. IEEE International Symposium on a World of Wireless, Mobile and Multimedia Networks (WoWMoM), Jun 2011, pp. 1-6.

[11] J. S. Jung et al., "Improving IEEE 802.11s wireless mesh networks for reliable routing in the smart grid infrastructure," in Proc. IEEE International Conference on Communications Workshops (ICC), Jun 2011, pp. 1-5.

[12] H. Gharavi and B. Hu, "Multigate mesh routing for smart grid last mile communications," in Proc. IEEE Wireless Communications and Networking Conference (WCNC), March 2011, pp. 275-280.

[13] — "Multigate communication network for smart grid," in Proceedings of the IEEE, vol. 99, no. 6, Jun 2011, pp. 1028 - 1045.

[14] G. Rajalingham, Q.-D. Ho, and T. Le-Ngoc, "Attainable throughput, delay and scalability for geographic routing on smart grid neighbor area networks," in IEEE Wireless Communications and Networking Conference (WCNC'2013), Apr. 2013.

[15] _ "Evaluation of an efficient smart grid communication system at the neighbor area level," in the 11th Annual IEEE Consumer Communications \& Networking Conference (CCNC'2014), Jan. 2014.

[16] Q.-D. Ho, Y. Gao, G. Rajalingham, and T. Le-Ngoc, "Performance and applicability of candidate routing protocols for smart grid's wireless mesh neighbor-area networks," in IEEE International Conference on Communications (ICC'2014), Jun. 2014.

[17] A. M. Popescua et al., "Surveying position based routing protocols for wireless sensor and ad-hoc networks," International Journal of Communication Networks and Information Security (IJCNIS), vol. 4, no. 1, pp. 41-67, Apr 2012.

[18] B. Karp and H. Kung, "GPSR: greedy perimeter stateless routing for wireless networks," in Proc. Annual International Conference on Mobile Computing and Networking (MobiCom), Boston, MA, USA, Aug 2000, pp. 243-254

[19] T. Watteyne et al., "From MANET to IETF ROLL standardization: A paradigm shift in WSN routing protocols," IEEE Communications Surveys \& Tutorials, vol. 13, no. 4, pp. 688-707, Jan 2011.

[20] NIST Priority Action Plan 2 - Guidelines for Assessing Wireless Standards for Smart Grid Applications, National Institude of Standards and Technology Std., 2011.

[21] D. Laverty, D. Morrow, R. Best, and P. Crossley, "Telecommunications for smart grid: Backhaul solutions for the distribution network," in Power and Energy Society General Meeting, 2010 IEEE, July 2010, pp. 1 -6.

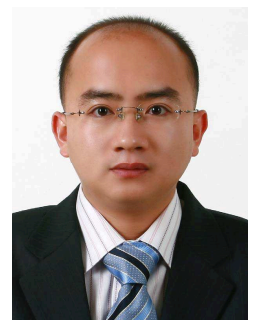

Quang-Dung Ho received his B.Eng. in Electrical Engineering and B.Sc. in Computer Science from Hochiminh City University of Technology (Vietnam) in May 2000 and Hochiminh City University of Natural Sciences (Vietnam) in Sep. 2000, respectively. He then obtained his M.Sc. and Ph.D. in Electrical and Computer Engineering from Information and Communications University (South Korea) in Feb. 2003 and Korea Advanced Institute of Science and Technology (South Korea) in Feb. 2007, respectively. From May 2000 to May 2001, he joined the Electrical Engineering Department, Hochiminh City University of Technology (Vietnam) as a Lecturer. In 2002, he worked as an Invited Researcher for Intelligence Inc., Daedeok Science Valley (South Korea). In 2006, he worked as a Research Engineer in Global Technology Team, SK Telecom (South Korea). From 2007 to present, he has been working as a Post-Doctoral Fellow and then Research Associate in Broadband Communications Lab, Electrical and Computer Engineering Department, McGill University (Canada). His current main research interests include dynamic routing and resource allocation in wireless networks, cloud computing and wireless virtualization. Besides, he has been designing and implementing network simulators, experimental platforms and real-life products for electronic healthcare, smart grid and next-generation wireless networks.

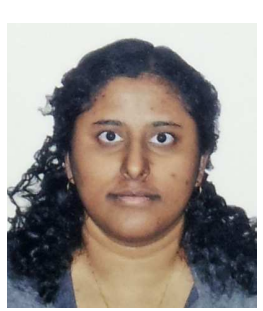

Gowdemy Rajalingham received her B.Eng. in Electrical Engineering from McGill University (Canada) in February 2012. She is currently working towards her M.Eng. at McGill University (Canada). From 2010 to present she has been working as a research assistant in the Broadband Communications Lab, Electrical and Computer Engineering Department, McGill University (Canada). Her current main research interests include network coding and network routing in wireless networks, cloud computing and wireless virtualization. She is involved in designing and implementing wireless network routing protocols both in simulation platforms and embedded devices for smart grid and smart health applications.

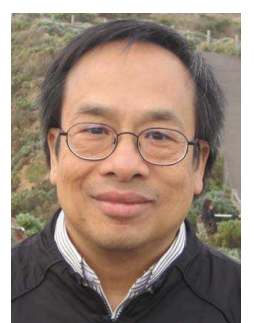

Tho Le-Ngoc is a Professor with the Department of Electrical and Computer Engineering, McGill University, Montreal, QC, Canada. He received the B.Eng. (with Distinction) degree in electrical engineering in 1976, the M.Eng. degree in microprocessor applications in 1978 from McGill University, Montreal, QC, Canada, and the Ph.D. degree in digital communications in 1983 from the University of Ottawa, ON, Canada. His research interest is in the area of broadband digital communications. Dr. Le-Ngoc is a Fellow of the Royal Society of Canada, the Institute of Electrical and Electronics Engineers, the Engineering Institute of Canada, and the Canadian Academy of Engineering. He is a Senior Member of the Ordre des Ingenieurs du Quebec. He is the recipient of the 2004 Canadian Award in Telecommunications Research, and the 2005 IEEE Canada Fessenden Award. He is the Canada Research Chair (Tier I) on Broadband Access Communications and the Bell Canada/NSERC Industrial Research Chair on Performance \& Resource Management in Broadband xDSL Access Networks. 